\title{
Short-Term Retention of Paired Associates as a Function of Instructions and Retention Measure ${ }^{1}$
}

\author{
MiChele H. GARSKOF ${ }^{2}$ \\ University of Michigan, Ann Arbor, Michigan 48104
}

\begin{abstract}
Two sets of instructions (traditional paired-associate instructions and special instructions to mediate) and three retention tasks (free recall, modified free recall, and recognition matching) were used to investigate the unlearning of first-list associates when an A-B, A-C paradigm was used in a short-term memory (STM) situation. Experimental Ss were given $2 \mathrm{sec}$ per pair to learn two A-B pairs and two A-C pairs and then $10 \mathrm{sec}$ of interpolated number reading before a retention test was administered. Control $S$ s learned only List-1 pairs and had additional interpolated material to equate retention interval. Performance was poorer for the experimental group than the control group in all conditions except with mediating instructions and the recognition-matching task. These results provide evidence for unlearning in STM and provide further support for a two-stage model of unlearning.
\end{abstract}

Underwood, Runquist and Schulz (1959) have described paired-associate (PA) learning as a two-stage process consisting of a responselearning stage and an associative hook-up stage. The former consists of learning the responses in the list, while the latter consists of attaching each response to the appropriate stimulus. McGovern (1964) suggested that two analogous processes may occur in unlearning. During List-2 acquisition, List-1 responses may be extinguished, or they may remain available but become no longer associatively connected with the appropriate stimuli. Another possibility is that the responses become unavailable while the associative connections remain intact. McGovern (1964), and Garskof and Sandak (1964) used a recognition-matching task ( $R M)$ to test the availability of associative connections inde-

1 This research was supported by the Advanced Research Projects Agency, Department of Defense, and was monitored by the Air Force Office of Scientific Research, under Contract No. AF 49(638)-1235 with the Human Performance Center, Department of Psychology.

${ }^{2}$ The author wishes to thank Judith Goggin for her thoughtful advice and her careful reading of the manuscript. pendently from the availability of responses. Stimuli and responses were made available and $S$ s were asked to match all the pairs from both lists. Since response terms were given, extinction of responses could not affect performance. Using an A-B, A-C paradigm (same stimuli, unrelated responses), both McGovern, and Garskof and Sandak found significant decrements in List-1 associations with RM. This was evidence for unlearning of associative connections.

Garskof, Sandak, and Malinowski (1965) used traditional PA instructions (TI) and special instructions that asked $S$ s to use mnemonic devices to form associations between stimuli and responses. The mediating instructions (MI) resulted in faster learning for both RM and MMFR groups, but only with the RM task were there significantly more correct List-1 responses with mediation. The fact that MI increased retention with the RM task, but not with the MMFR task, indicated that the two processes of unlearning could be independently manipulated. In this case, MI enhanced the retention of the associative hook-up, but did not affect response unlearning, so that only when the responses were made 
available did $S$ s perform better on the retention test.

As a test of the generality of the interference theory of forgetting, Melton (1963) has called for an examination of its ability to predict forgetting in short-term memory (STM). The purpose of the present study was to investigate the unlearning of first-list associates in a STM situation, and to determine the effects of special mediating instructions on the two stages of unlearning in STM.

Two sets of instructions were used, MI which asked $S$ s to use mnemonic devices to form associations between stimulus and response terms, and traditional PA instructions. Three retention tasks were used: free recall (FR), MMFR, and RM. FR required $S$ s to write all the response terms they remembered, in any order.

It was expected that the mediating instructions would increase the level of learning (as in Garskof et al., 1965), especially for the A-C pairs, since the A-B pairs are learned very well with traditional instructions (Goggin, 1966). It was also predicted that these instructions would affect the associative hook-up stage of learning rather than the response-learning stage. Therefore, retention would be greater for $S$ s receiving mediating instructions and the RM task than for those who received traditional instructions and the RM task. FR and MMFR would not be affected by the mediating instructions.

\section{MetHoD}

Subjects. The Ss were 162 students from introductory psychology classes. Participation in this experiment helped to fulfill a course requirement. There were $18 S \mathrm{~s}$ in each experimental condition, and nine in each control condition. The $S s$ were assigned to conditions in order of appearance in the laboratory according to a predetermined sequence of random assignment.

Design. Independent variables were type of instructions (MI and $\mathrm{TI}$ ), and retention tasks (FR, MMFR, and RM). There were 12 groups: six experimental groups and six control groups (in each case, two types of instructions $\times$ three retention tasks). Each $S$ received three replications of the experiment in the condition to which he was randomly assigned. The A-B, A-C paradigm was used in the experimental conditions. List 1 consisted of two A-B pairs, and List 2 consisted of two A-C pairs.

Experimental $S s$ were presented with six A-B pairs, two for each test, and six $C$ responses. There was one set of these materials, with two different pairings. Each pairing was used nine times in each experimental condition. Three different sets of two-pair subgroups were used for each pairing, and each of these subgroups served equally often as Tests 1,2 , and 3 . The subgroups always followed each other in the same order so that sequence effects were not controlled.

The experimental groups in the MMFR and RM conditions were presented with the stimulus terms four times: once each in Lists 1 and 2, and twice in the retention test. Functionally, however, there were only three repetitions since the occurrences of an individual stimulus were consecutive in the retention test. Two of the eight possible orders of presentation were excluded; considering Stimulus 1 as ' $a$ ' and Stimulus 2 as ' $b$ ' these were: ababab and bababa. The other six orders were used equally often in each condition and in each test.

Three random orders of the four response terms were used in the RM task. Each of the following was assigned once to each of the six orders of the stimulus terms appearance: $B_{2} C_{1} C_{2} B_{1}, C_{1} B_{2} C_{2} B_{1}$, and $C_{2} B_{1} B_{2} C_{1}$.

Control Ss received only one list but the time between List 1 presentation and the retention task was made equal to that in the experimental conditions by the inclusion of additional interpolated activity. The control groups in the MMFR and RM conditions were presented with the stimulus terms once in List 1 and once in the retention test. The following orders were each used half of the time (either four or five times per condition): abba and abab.

Materials. Stimulus terms were CVC's with association values of $93 \%$ (Glaze, 1928). Of the six stimulus terms, no two had the same first letters.

Response terms were six-letter, two-syllable adjectives with AA frequency of occurrence in the language (Thorndike and Lorge, 1944). All apparent associative connection between response items were minimized by inspection.

Procedure. The materials were presented serially at a 2-sec rate. The stimulus and response of a pair were shown simultaneously. For the interpolated activity, Ss read aloud, one by one, as fast as possible, five 8-digit series (seven series in the control conditions). Each 8-digit series was presented for $2 \mathrm{sec}$. As soon as the interpolated activity was completed, a row of asterisks appeared on the memory drum and the appropriate retention instructions were begun. A 30 -sec interval was employed for the retention task. 
Tests 2 and 3 were begun immediately after the 30-sec period of the previous test, except in the FR conditions where $S$ s were asked to pair stimuli and responses. On the average, $5 \mathrm{sec}$ were taken to make these pairings. At the conclusion of the experiment, $S$ s were questioned informally about the mediators they used.

The TI instructions directed $S$ s to learn the pairs, whereas the MI instructions included an additional paragraph asking $S$ s to form meaningful associations between the stimulus and response terms. Two trials were administered with a practice tape on which the CVC's and words were represented by lines. The numbers were included, however, and $S$ s read them aloud for practice.

Each test consisted of showing once Lists 1 and 2, followed by the interpolated activity of number reading. Then the appropriate retention materials were presented and retention instructions administered. The administration of these instructions took $2 \mathrm{sec}$.

\section{RESULTS AND DISCUSSION}

Two types of scoring methods were used with the MMFR and FR conditions. Lenient scoring considered a response correct if it was given, whether or not it was paired with the correct stimulus. With stringent scoring, a response was considered correct if it was given and attached to the correct stimulus. Only stringent scoring was applied to the RM conditions. A square-root transformation on the retention scores preceded each analysis.

Table 1 presents the results of Test 1 . It can be seen that (a) performance was high for control $S \mathrm{~s}$; (b) performance was poorer in the A-B, A-C paradigm than the control in all conditions except RM with $\mathrm{MI}$; (c) recall in the MMFR and FR conditions was equivalent as long as the same method of scoring was applied to both, stringent scoring yielding lower scores than lenient scoring. To avoid repetition, stringent MMFR scores and lenient FR scores were used in all the subsequent analyses.

Three $2 \times 2$ analyses of variance (Control/ Experimental $\times$ Instructions) were performed on the Test-1 data, one for each retention test. It is evident from Table 1 that no significant differences existed between MMFR and FR scores, and Bahrick and Bahrick (1964) provide documented arguments for not considering the comparisons between recognition and
TABLE 1

Mean Correct Responses on Test 1

\begin{tabular}{|c|c|c|c|c|}
\hline & \multicolumn{2}{|c|}{$\mathrm{A}-\mathrm{B}, \mathrm{A}-\mathrm{C}$} & \multicolumn{2}{|c|}{ Control } \\
\hline & Str. & Len. & Str. & Len. \\
\hline \multicolumn{5}{|l|}{ MMFR } \\
\hline $\mathrm{TI}$ & 1.11 & 1.33 & 1.67 & 1.89 \\
\hline MI & 1.22 & 1.38 & 1.78 & 1.78 \\
\hline \multicolumn{5}{|l|}{ FR } \\
\hline TI & 1.11 & 1.39 & 1.78 & 1.89 \\
\hline MI & 1.11 & 1.33 & 1.67 & 1.78 \\
\hline \multicolumn{5}{|l|}{$\mathbf{R M}$} \\
\hline TI & 1.00 & - & 1.79 & - \\
\hline MI & 1.83 & - & 1.78 & - \\
\hline
\end{tabular}

recall tasks. With the MMFR task, the control groups performed significantly better than the experimental groups, $F(1,50)=7.47, p<0.01$. There was no significant effect of instructions, $F(1,50)=0.33, p>0.05$, and the interaction of control/experimental with type of instructions was nonsignificant, $F(1,50)=1.85$, $p>0.05$. With the FR task the findings were similar. There was a significant control/ experimental effect, $F(1,50)=8.41, p<0.01$. Instruction effects, $F<1$, and the interaction of these two variables, $F=0.00$, were nonsignificant. With the RM task, ${ }^{3}$ the control/ experimental difference was nonsignificant, $F(1,50)-3.06, p>0.05$. MI lcd to grcater retention than did TI, $F(1,50)=5.02, p<0.05$, and there was a significant interaction, $F(1$, $50)=5.02, p<0.05$. This reflects the fact that with TI the control group had greater retention than the experimental group (as with the MMFR and FR tasks), but with MI retention was greatly improved in the experimental condition.

${ }^{3}$ It is important to note that only List-1 performance affected $S$ 's score, regardless of the condition to which $S$ was assigned. Because of this, comparisons between experimental and control groups with the RM task are not biased by differences in the number of responses to be matched. In either the experimental or control condition if responses are randomly assigned to stimuli, the expected score is 1.00 . 
Since significantly fewer first-list responses were retained after A-C learning than in the control conditions, it appears that unlearning did take place in all the TI conditions and in MI conditions of MMFR and FR. With the RM task, however, MI had a significant effect; A-B retention was not interfered with by A-C learning.

Misplaced responses made up more than $90 \%$ of the errors. However, the RM task limits the type of errors to only misplaced responses since the correct stimulus and response terms are supplied.

Tests 2 and 3 were used to measure cumulative proactive inhibition employing the method adopted by Goggin (1966). For none of the retention measures was a decrease in retention statistically significant.

These results confirm the findings of Garsk of et al. (1965), and in doing so add to the plausibility of two stages in the unlearning process. The MI instructions seem to enhance the hookup stage. It is possible that MI makes List-1 associations resistant to retroactive inhibition because $S$, in following the instructions to find a mediator, encodes the pair into a single to-beremembered unit. This would result in fewer interfering units.

Apparently, the advantage of MI can be reflected only by the matching task, in which the responses that have been extinguished during A-C learning are made available to $S$. This may be because RM enables $S$ to use backward associations. Degree of R-S learning increases with the degree of S-R learning (Jantz and Underwood, 1958) and since MI has been shown to increase the degree of S-R learning (Garskof et al., 1965), MI should also produce greater $\mathrm{R}-\mathrm{S}$ learning. Since $\mathrm{RM}$ gives $S$ opportunity to use both backward and forward associations, RM with MI should yield better performance than RM with TI.

\section{REFERENCES}

BaHrick, H. P., AND Bahrick, P. O. A re-examination of the interrelations among measures of retention. Quart.J. exp. Psychol., 1964, 16, 318-324.

BARnes, J. M., AND UNDERwoOd, B. J. "Fate" of first list associations in transfer theory.J.exp. Psychol., 1959, 58, 97-105.

GarsKof, B. E., AND SANDAK, J. M. Unlearning in recognition memory. Psychon. Sci., 1964, 1, 197198.

Garskof, B. E., Sandak, J. M., and Malinowski, E. W. A. Controlling the "fate" of first list associates. Psychon. Sci., 1965, 2, 315-316.

Glaze, J. A. The association value of nonsense syllables. J. genet. Psychol., 1928, 35, 255-267.

Gogarn, J. Proactive and retroactive inhibition in the short-term retention of paired associates. J. verb. Learn. verb. Behav., 1966, 5, 526-535.

JANTZ, E. M., AND UNDERWOOD, B. J. R-S learning as a function of meaningfulness and degree of S-R learning. J. exp. Psychol., 1958, 56, 174-179.

MCGovern, J. B. Extinction of associations in four transfer paradigms. Psychol. Monogr., 1964, 78 (16 Whole No. 593).

Melton, A. W. Implications of short-term memory for a general theory of memory. J. verb. Learn. verb. Behav., 1963, 2, 1-21.

THORNDIKE, E. L., AND LORGE, I. The teacher's word book of 30,000 words. New York: Teachers College, Columbia University, 1944.

UNDERWOOD, B. J., RUNQUIST, W. W., AND SCHULz, R. W. Response learning in paired-associate lists as a function of intra-list similarity. $J$. exp. Psychol., 1959, 58, 70-78.

(Received January 20, 1967) 